\title{
da Vinci robot-assisted keyhole neurosurgery: a cadaver study on feasibility and safety
}

\author{
Hani J. Marcus • Archie Hughes-Hallett • Thomas P. Cundy • \\ Guang-Zhong Yang • Ara Darzi • Dipankar Nandi
}

Received: 12 May 2014 / Revised: 28 September 2014 / Accepted: 10 October 2014 / Published online: 18 December 2014

(C) The Author(s) 2014. This article is published with open access at Springerlink.com

\begin{abstract}
The goal of this cadaver study was to evaluate the feasibility and safety of da Vinci robot-assisted keyhole neurosurgery. Several keyhole craniotomies were fashioned including supraorbital subfrontal, retrosigmoid and supracerebellar infratentorial. In each case, a simple durotomy was performed, and the flap was retracted. The da Vinci surgical system was then used to perform arachnoid dissection towards the deep-seated intracranial cisterns. It was not possible to simultaneously pass the $12-\mathrm{mm}$ endoscope and instruments through the keyhole craniotomy in any of the approaches performed, limiting visualization. The articulated instruments provided greater dexterity than existing tools, but the instrument arms could not be placed in parallel through the keyhole craniotomy and, therefore, could not be advanced to the deep cisterns without significant clashing. The da Vinci console offered considerable ergonomic advantages over the existing operating room arrangement, allowing the operating surgeon to remain non-sterile and seated comfortably throughout the procedure. However, the lack of haptic feedback was a notable limitation. In conclusion, while robotic platforms have the potential to greatly enhance the performance of transcranial approaches, there is strong justification for research into next-generation robots, better suited to keyhole neurosurgery.
\end{abstract}

Keywords Image guided intervention $\cdot$ Minimally invasive surgery $\cdot$ Neurosurgery $\cdot$ Robotic surgery

H. J. Marcus · A. Hughes-Hallett · T. P. Cundy · G.-Z. Yang •

A. Darzi

The Hamlyn Centre for Robotic Surgery, Institute of Global Health Innovation, Imperial College London, Paterson Building (Level 3), Praed Street, London W2 1NY, UK

H. J. Marcus $(\bowtie) \cdot D$. Nandi

Department of Neurosurgery, Imperial College Healthcare NHS

Trust, London, UK

e-mail: hani.marcus10@imperial.ac.uk

\section{Introduction}

Robotic platforms that further enhance surgical skills have the potential to improve the safety and efficacy of keyhole neurosurgery $[9,11,22]$. At present, the most widely used surgical robot worldwide is the da Vinci system (Intuitive Surgical, CA, USA), a master-slave system designed for minimally invasive surgery (MIS) in which the surgeon remotely controls the robots' actions (Fig. 1). The camera arm includes two lenses, providing surgeons with a high-resolution stereoscopic image of the operative field. The instrument arms contain articulated endo-wrists, which increase surgical dexterity. In addition, the system allows for motion scaling and tremor filtering. To date, the da Vinci system has been used in a broad range of procedures $[2,12]$ and has achieved substantial clinical penetration in fields such as urology $[5,13]$.

Recently, a group has reported the application of the da Vinci surgical system to assist with the supraorbital subfrontal approach through an eyebrow skin incision in a cadaver study [6]. While the authors concluded that such robot-assisted approaches were probably feasible, they noted several drawbacks including the lack of suitable instruments such as bone cutters and the risk of arm collisions and highlighted the need for further studies. The goal of the present cadaver study was therefore to confirm and extend these preliminary findings by applying the da Vinci surgical system to a range of keyhole neurosurgical approaches.

\section{Methods}

Ethical approval was obtained from the Proportionate Review Sub-Committee of the National Research Ethics Service (NRES) Committee East Midlands. A formalin-fixed cadaver head was obtained from the Department of Anatomy, Imperial College London. Surgical residents (HJM and AHH) 

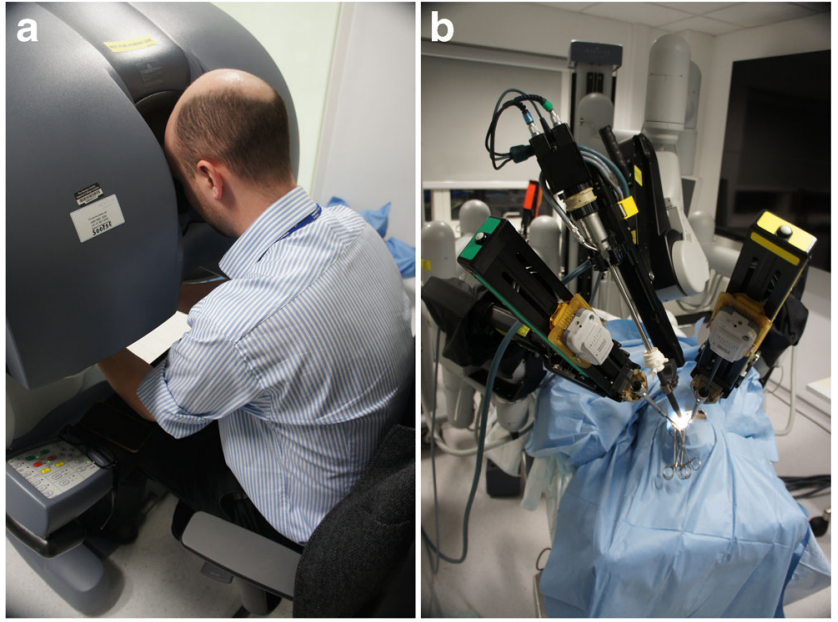

Fig. 1 Arrangement of the da Vinci master-slave system. a The surgeon is seated comfortably at the console and remotely controls the robots' actions. b The surgical cart includes an endoscope and instrument arms and carries out the procedure. Note the difficulty in parallel insertion of the instrument arms through a single keyhole craniotomy

performed the procedures under the supervision of the senior clinical authors (DN and $\mathrm{AD}$ ), who are experienced in minimally invasive neurosurgery and robot-assisted surgery, respectively.

The cadaver head was secured in a Mayfield clamp and a Budde Halo retractor system attached (Integra, NJ, USA). An Albert Wetzlar operating microscope (Albert Wetzlar GmbH, Wetzlar, Germany) and high-speed drill (B. Braun, Melsungen, Germany) were used to fashion several previously described keyhole craniotomies, approximately $20-30 \mathrm{~mm}$ in diameter $[15,17]$ : supraorbital subfrontal, retrosigmoid and supracerebellar infratentorial. Craniotomies were not extended to accommodate the da Vinci system; instead, the aim was to determine the suitability of the robot to typical keyhole craniotomies. In each approach, a simple durotomy was performed, and the flap was retracted.

Fig. 2 Left supraorbital subfrontal approach through an eyebrow incision demonstrating the following: a keyhole craniotomy approximately $25 \times 15 \mathrm{~mm}$ in size, $\mathbf{b}$ a $12-\mathrm{mm}$ endoscope and two standard 8$\mathrm{mm}$ instruments were unable to enter the keyhole simultaneously, c a 12-mm endoscope and two smaller 5-mm instruments were also unable to enter the keyhole simultaneously, and $\mathbf{d}$ endoscopic visualization was therefore limited
The standard da Vinci robotic system was used intradurally, and arachnoid dissection was performed towards the deepseated cisterns. The surgeon remained non-sterile at the robot console, while an assistant was available throughout the procedure to exchange robotic instruments. Both $0^{\circ}$ and $30^{\circ}$ upwards facing 12-mm endoscopes were introduced into the keyhole craniotomy for visualization. Standard 8-mm and smaller 5-mm instruments were used for tissue manipulation. Throughout each procedure, a detailed feedback was obtained, including images of the operating room arrangement and of the endoscope feed.

\section{Results}

It was not possible to simultaneously pass the 12 -mm endoscope and instruments through the keyhole craniotomy in any of the approaches performed, irrespective of whether two standard 8-mm instruments or smaller 5-mm instruments were used (Fig. 2). Instead, the endoscope had to be placed outside the craniotomy, limiting the illumination, magnification, and wide-angle view provided.

Dissection with the da Vinci instruments was restricted to superficial structures, approximately $20 \mathrm{~mm}$ from the craniotomy. The large instrument arms could not be placed in parallel through the keyhole craniotomy and, therefore, could not be advanced to the deep cisterns without significant clashing (Fig. 1). The smaller 5-mm instruments were comparatively easier to pass through the keyhole craniotomy but utilized tentacle-like continuum tool shafts rather than the articulated wrist joints that characterize standard $8-\mathrm{mm}$ instruments. The result, paradoxically, was that the smaller $5-\mathrm{mm}$ instruments had less dexterity than the standard 8-mm instruments in the spatially constrained intracranial cisterns (Fig. 3). The range of 5-mm instruments was also limited, with no bipolar forceps or
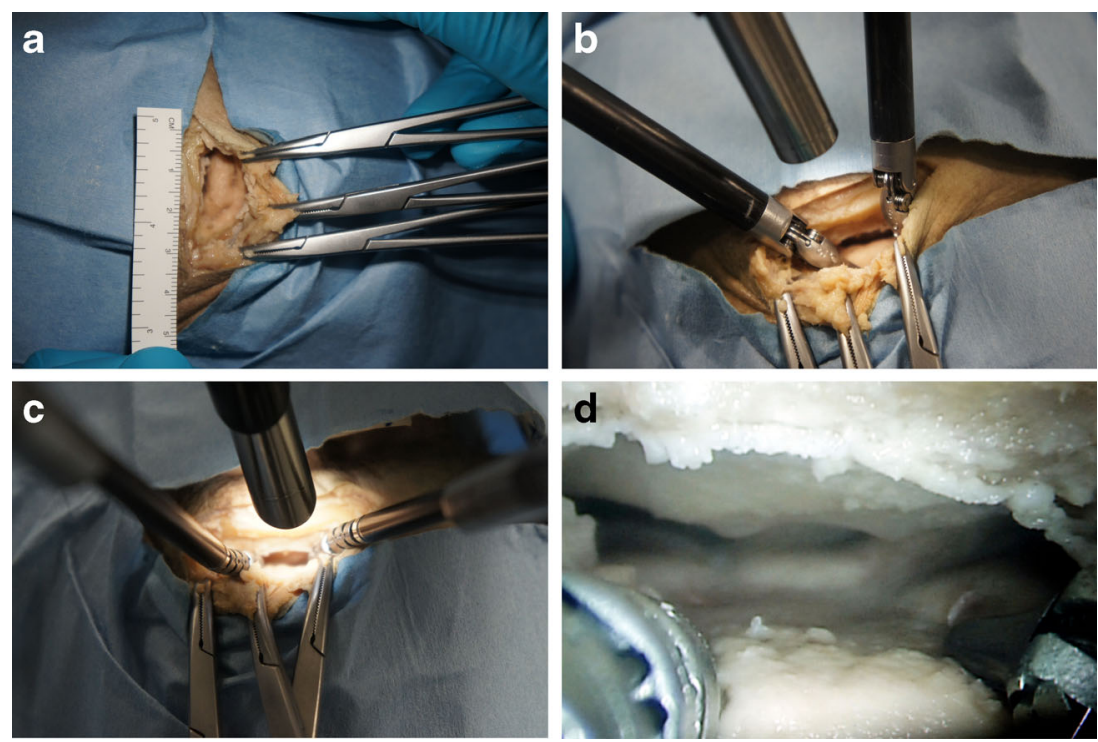
Fig. 3 Comparison of a 8-mm da Vinci instruments with articulated wrist joints and (b) 5-mm da Vinci instruments with tentaclelike continuum tool shafts
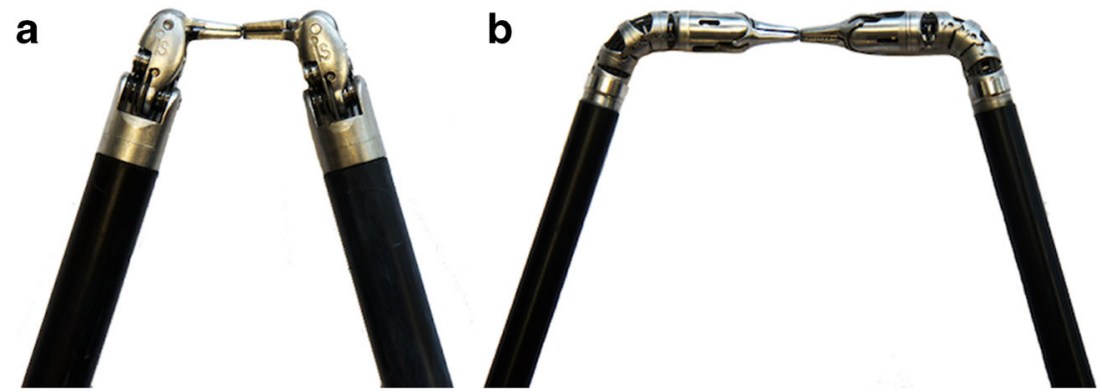

suction-irrigation available. Nonetheless, all the robotic instruments used during the study allowed for greater dexterity than existing rigid tube shaft instruments.

The da Vinci console offered considerable ergonomic advantages over the existing operating room arrangement, allowing the operating surgeon to remain non-sterile and seated comfortably throughout the procedure (Fig. 2). The visualization provided by the $3-\mathrm{D}$ endoscope provided an immersive view of the operative field. Control of the instruments was intuitive and allowed for motion scaling and tremor filtering. However, the lack of haptic feedback was a notable limitation.

\section{Discussion}

In this cadaver study, it has been demonstrated that use of the standard da Vinci robotic system in keyhole transcranial endoscope-assisted microsurgery is neither safe nor feasible. Arguably, the greatest role of surgical robots is as a "great leveler", allowing surgeons to perform keyhole approaches when they would otherwise resort to open surgery [10]. To this end, technically challenging keyhole neurosurgical approaches are ideal targets for such surgical robots. Regrettably, in its present form, the da Vinci robotic platform is ill-suited to brain surgery given its multiple large and bulky arms, limited selection of instruments and lack of haptic feedback.

In a related study, Thakre et al. sought to evaluate the performance of the da Vinci platform in increasingly small workspaces [18]. It was noted that a cube had to be at least $40 \mathrm{~mm}$ in size to simultaneously pass both the endoscope and instruments, at least $50 \mathrm{~mm}$ in size to perform standard surgical drills and at least $60 \mathrm{~mm}$ in size to do so without significant collision between the instrument arms (albeit with difficulty). These findings broadly corroborate those of the present study, in which it was not possible to simultaneously pass both the endoscope and instruments through keyhole craniotomies approximately $20-30 \mathrm{~mm}$, and instrument clashes prevented dissection towards the deep intracranial cisterns (see Table 1).

The present cadaver study has several inherent limitations. The cadaver brain was formalin-fixed and was therefore not as a compliant as the living brain, though pathology resulting in cerebral oedema can be similarly tense. The cadaver brain also did not allow for assessment of haemostasis, which is frequently cited as a limitation of keyhole approaches. Animal studies might better address the issue of haemostasis, but there are very few animal models with brains of a similar size to humans; such studies would be logistically difficult to organize and also raise ethical concerns.

While several studies have reported the use of the da Vinci robot in spinal and peripheral nerve surgery $[1,4,7,14,16$, 19-21], only one previous cadaver study has described the use of the da Vinci robot in keyhole brain surgery [6]. Hong et al. described the application of the da Vinci robot to the keyhole supraorbital approach and, in contrast to the present study, found that it was generally feasible, though they did comment on instrument clashes and lack of proper tools. We speculate that this discrepancy may be due to their longer incision and larger craniotomy and their use of brain retractors.

Recently, a group from the University of Washington has proposed new multiport approaches to the anterior cranial fossa that are better suited to the da Vinci robotic platform
Table 1 Summary of the limitations of the da Vinci platform in small working spaces using data from the present cadaver study (size $<40 \mathrm{~mm}$ ) and the previous preclinical study by Thakre et al. (size $\geq 40 \mathrm{~mm}$ )

Previous preclinical study by Thakre et al. [18]

\begin{tabular}{lllll}
\hline & Size (mm) & $\begin{array}{l}\text { Visualization with } \\
\text { endoscope }\end{array}$ & $\begin{array}{l}\text { Manipulation with 8-mm } \\
\text { instruments }\end{array}$ & Immersive console \\
\hline Keyhole craniotomy & 20 & Limited & Limited & Full \\
& 30 & Limited & Limited & Full \\
& 40 & Full & Limited & Full \\
& 50 & Full & $\begin{array}{l}\text { Full but with instrument } \\
\text { collisions }\end{array}$ & Full \\
& 60 & Full & $\begin{array}{c}\text { Full without collisions, } \\
\text { but with difficulty }\end{array}$ & Full \\
Minicraniotomy & 70 or greater & Full & Full & Full \\
\hline
\end{tabular}


[3]. In order to overcome the narrow funnel effect generated from arms in close proximity, and the steep angle of approach to the skull base, they suggest transnasal and bilateral medial orbital ports for the camera and instruments, respectively. While such novel approaches may be more viable from a technical standpoint, they carry a greater risk of approachrelated morbidity than standard keyhole approaches.

Alongside the technical challenges to the use of the da Vinci robotic platform in keyhole neurosurgery highlighted in the present study, several other barriers also exist [8]. Arguably, the greatest drawback to existing surgical robots is economic. The current da Vinci robot, for example, is priced at over $\$ 2 \mathrm{~m}$, carries substantial maintenance costs and requires additional training of the surgeons and nurses involved with its use. Next-generation robotic platforms may mitigate these limitations. Over time, the large and expensive multipurpose robots of today are likely to be replaced by smaller and more affordable robots tailored to particular procedures [8].

\section{Conclusions}

Keyhole transcranial endoscope-assisted microsurgical techniques are technically challenging approaches that may greatly benefit from surgical robotics. However, the most widely used surgical robot worldwide today, the da Vinci platform, is neither safe nor feasible to use in keyhole neurosurgery. There is therefore strong justification for research into nextgeneration robots, better suited to such approaches.

Acknowledgments H. J. Marcus is supported by an Imperial College Wellcome Trust Clinical Fellowship.

Open Access This article is distributed under the terms of the Creative Commons Attribution License which permits any use, distribution, and reproduction in any medium, provided the original author(s) and the source are credited.

\section{References}

1. Aaronson OS, Tulipan NB, Cywes R, Sundell HW, Davis GH, Bruner JP, Richards WO (2002) Robot-assisted endoscopic intrauterine myelomeningocele repair: a feasibility study. Pediatr Neurosurg 36(2):85-89. doi:10.1159/000048358

2. Allison N, Tieu K, Snyder B, Pigazzi A, Wilson E (2012) Technical feasibility of robot-assisted ventral hernia repair. World J Surg 36(2): 447-452. doi:10.1007/s00268-011-1389-8

3. Bly RA, Su D, Lendvay TS, Friedman D, Hannaford B, Ferreira M, Moe KS (2013) Multiportal robotic access to the anterior cranial fossa: a surgical and engineering feasibility study. Otolaryngol Head Neck Surg 149(6):940-946. doi:10.1177/0194599813509587
4. Deboudt C, Labat JJ, Riant T, Bouchot O, Robert R, Rigaud J (2012) Pelvic Schwannoma: robotic laparoscopic resection. Neurosurgery. doi:10.1227/NEU.0b013e31826e2d00

5. Hofer MD, Meeks JJ, Cashy J, Kundu S, Zhao LC (2013) Impact of increasing prevalence of minimally invasive prostatectomy on open prostatectomy observed in the national inpatient sample and national surgical quality improvement program. J Endourol 27(1):102-107. doi:10.1089/end.2012.0315

6. Hong WC, Tsai JC, Chang SD, Sorger JM (2013) Robotic skull base surgery via supraorbital keyhole approach: a cadaveric study. Neurosurgery 72(Suppl 1):33-38. doi:10.1227/NEU. 0b013e318270d9de

7. Lee JY, Lega B, Bhowmick D, Newman JG, O'Malley BW Jr, Weinstein GS, Grady MS, Welch WC (2010) Da Vinci Robotassisted transoral odontoidectomy for basilar invagination. ORL J Otorhinolaryngol Relat Spec 72(2):91-95. doi:10.1159/000278256

8. Marcus H, Nandi D, Darzi A, Yang GZ (2013) Surgical robotics through a keyhole: from today's translational barriers to tomorrow's “disappearing" robots. IEEE Trans Biomed Eng 60(3):674-681. doi: 10.1109/TBME.2013.2243731

9. Marcus HJ, Cundy TP, Hughes-Hallett A, Yang GZ, Darzi A, Nandi D (2014) Endoscopic and keyhole endoscope-assisted neurosurgical approaches: a qualitative survey on technical challenges and technological solutions. Br J Neurosurg. doi:10.3109/02688697.2014. 887654

10. Marcus HJ, Hughes-Hallett A, Cundy TP, Nandi D, Yang GZ, Darzi A (2013) Not everything that counts can be easily counted. BMJ 346: f2461. doi:10.1136/bmj.f2461

11. Marcus HJ, Seneci CA, Payne CJ, Nandi D, Darzi A, Yang GZ (2014) Robotics in keyhole transcranial endoscope-assisted microsurgery: a critical review of existing systems and proposed specifications for new robotic platforms. Neurosurgery 10(Suppl 1):84-95. doi:10.1227/NEU.0000000000000123, discussion 95-86

12. Modi P, Rodriguez E, Chitwood WR Jr (2009) Robot-assisted cardiac surgery. Interact Cardiovasc Thorac Surg 9(3):500-505. doi:10.1510/ icvts.2009.203182

13. Patel VR, Thaly R, Shah K (2007) Robotic radical prostatectomy: outcomes of 500 cases. BJU Int 99(5):1109-1112. doi:10.1111/j. 1464-410X.2007.06762.X

14. Perez-Cruet MJ, Welsh RJ, Hussain NS, Begun EM, Lin J, Park P (2012) Use of the da Vinci minimally invasive robotic system for resection of a complicated paraspinal schwannoma with thoracic extension: case report. Neurosurgery 71(1 Suppl Operative): onsE209-eE214. doi:10.1227/NEU.0b013e31826112d8

15. Perneczky A, Reisch R (2008) Keyhole approaches in neurosurgery, volume 1: concept and surgical technique, 1st edn. Springer, Vienna

16. Ponnusamy K, Chewning S, Mohr C (2009) Robotic approaches to the posterior spine. Spine (Phila Pa 1976) 34(19):2104-2109. doi:10. 1097/BRS.0b013e3181b20212

17. Reisch R, Marcus H, Koechlin N, Hugelshofer M, Stadie A, Kockro R (2013) Transcranial endoscope-assisted keyhole surgery: anterior fossa. Innov Neurosurg 1(2):77-89

18. Thakre AA, Bailly Y, Sun LW, Van Meer F, Yeung CK (2008) Is smaller workspace a limitation for robot performance in laparoscopy? J Urol 179(3):1138-1142. doi:10.1016/j.juro.2007.10.091, discussion 1142-1133

19. Yang MS, Kim KN, do Yoon H, Pennant W, Ha Y (2011) Robotassisted resection of paraspinal Schwannoma. J Korean Med Sci 26(1):150-153. doi:10.3346/jkms.2011.26.1.150

20. Yang MS, do Yoon H, Kim KN, Kim H, Yang JW, Yi S, Lee JY, Jung WJ, Rha KH, Ha Y (2011) Robot-assisted anterior lumbar interbody fusion in a Swine model in vivo test of the da vinci surgical-assisted spinal surgery system. Spine (Phila Pa 1976) 36(2):E139-E143. doi: 10.1097/BRS.0b013e3181d40ba3

21. Yang MS, Yoon TH, do Yoon H, Kim KN, Pennant W, Ha Y (2011) Robot-assisted transoral odontoidectomy: experiment in new 
minimally invasive technology, a cadaveric study. J Korean Neurosurg Soc 49(4):248-251. doi:10.3340/jkns.2011.49.4.248

22. Zada G, Liu C, Apuzzo ML (2013) "Through the looking glass": optical physics, issues, and the evolution of neuroendoscopy. World Neurosurg 79(2 Suppl):S3-S13. doi:10.1016/j.wneu.2013.02.001

\section{Comments}

\section{Ramez Kirollos, Cambridge, UK}

There is worldwide research into the future evolution of robotic or more precisely robot-assisted surgery. Nevertheless, such enthusiasm should be tempered by studies such as this cadaveric study by Marcus et al. exploring the feasibility and safety of using the da Vinci system for robot-assisted keyhole neurosurgery. This paper highlighted the need for improved system and instrument design tailored for specific approaches. The execution of microsurgical approaches adjacent to the deep basal cisterns not only depends on delicate maneuveres but also needs planning of safe trajectories to deep intracranial structures. These require adequate visualisation and safe manipulation of instruments. The current microsurgical techniques remain the gold standard, and further progress should prove safety and superiority over existing techniques prior to clinical application.

\section{Arya Nabavi, Hannover, Germany}

Marcus et al. describe their application of the da Vinci Robot for "keyhole" approaches in neurosurgery. They analyze several typical craniotomies in regard to the pure geometry of approach size vs robot needs. The smallest craniotomy is represented by a cube with $40-\mathrm{mm}$ base length. However, comfortable working was only possible with an opening of $60-\mathrm{mm}$ ("... without significant collision between the instrument arms (albeit with difficulty)"). Also, instruments and endoscope stand at a certain angle, which hinders working in the depth through small openings. They come to the drastic conclusion that "... the most widely used surgical robot worldwide today, the da Vinci platform, is neither safe nor feasible to use in keyhole neurosurgery" and ask for research into nextgeneration robots. The authors raise various important and crucial points and discuss them pointedly. However, the crucial concern which is raised by this article is how to analyze and report the functionality of new robotic tools.

Generally, papers on the da Vinci Robot are enthusiastic about the potential. Naturally, there are advantages to using a robot, be it ergonomics, better depth visualization or articulated instruments, which provide higher degrees of freedom. The limitations are mentioned more as an afterthought regarding haptics, limitations in confined spaces, referring to "...future work". This is understandable, since acquiring the system includes a significant amount of work (grants/ convincing) and money. Thus, most papers move to feasibility studies, which show that we, as neurosurgeons, can use almost any tool to reach our target. However, it does not provide us with information whether this is actually a step forward or an elaborate way to procrastinate or even regress, albeit with more sophisticated tools.

The initial da Vinci development was for laparoscopic surgeries. In those, it is crucial that instruments and endoscope stand at a certain angle, the instruments being inserted through separate skin incisions. Also, the existing spaces are much larger, the structures (e.g. bowels) less vulnerable to displacement. The advantages of this system are tailored to this surrounding. And, as always with expensive tools, the vendors strive to widen the user base. Neurosurgery is a very technology affine specialty, so it is natural for us to employ robotics. However, we should not lower our standards! The authors state that "Craniotomies were not extended to accommodate the da Vinci system; instead, the aim was to determine the suitability of the robot to typical keyhole craniotomies". This is the right approach to evaluating a new tool. Does it make us better, or do we have to compromise to use it? Do the compromises enhance our techniques or not? I am no advocate of constantly doing the same thing, but we have reached a very high standard, and we should not readily relinquish it, just to accommodate a new tool!

As we have developed standards for reporting in most areas of neurosurgery, we should be more specific about how we expect a robot to perform! What should be the core properties? What are we willing to relinquish for progress!?

At present, we have to concentrate our reports on a realistic representation of the advantages and a thorough analysis of the limitations of robotic systems, as in this paper. However, it is also our responsibility to articulate demands for the future development of robot assistance in neurosurgery. We must define our needs, or we will be confronted with numerous robot generations, which are feats of technological progress, but utterly useless in our field.

Elinor Warner, George Samandouras, London, UK

Marcus et al. from the Imperial College, London, UK, present a well-executed, technical feasibility study employing the da Vinci ${ }^{\circledR}$ robotic platform (Intuitive Surgical, CA, USA) to perform three keyhole approaches to a formalin-fixed cadaveric head: supraorbital subfrontal, retrosigmoid and supracerebellar infratentorial. They defined the maximum size of a keyhole craniotomy to 20 and $30 \mathrm{~mm}$ and attempted to insert a 12-mm endoscope and, in parallel, 8- and 5$\mathrm{mm}$ instruments, mounted, however, to much bulkier robotic arms. The result was, not surprisingly, instrument clash and operative inability to perform any meaningful or safe tissue dissection. The authors correctly concluded that the use of the da Vinci ${ }^{\circledR}$ robotic platform in keyhole transcranial approaches is, at present, neither feasible nor safe.

Neurosurgery is a technology-driven specialty that has been demanding mathematical and engineering breakthroughs during the last five decades to progress in advanced neuroimaging, reliable neuronavigation, modern, multifunctional operating room (OR) set-up and precise delivery of radiotherapy using Gamma Knife ${ }^{\circledR}$ and the robotic-based CyberKnife ${ }^{\circledR}$ platforms. In keeping with the rest of technology applications, evolution from working prototypes to seamlessly integrated, lowprofile functional components of the OR will take time, but possibly less time than one could estimate.

The prototype applications of robotic platforms have been the calculation of entry points and trajectory pathways used with three mainstream indications: stereotactic brain biopsies, stereotactic placement of brain electrodes for epilepsy and stereotactic placement of pedicle screw placement for scoliosis. These early applications have now entered routine clinical use in many centers. This month, in the Journal of Neurosurgery, a French group published a significant series of 100 consecutive frameless robotic stereotactic biopsies employing the ROSA ${ }^{\mathrm{TM}}$ (Medtech, Montpellier, France) robotic device (1). Many commercial and research groups continue to design and improve numerous robotic prototypes marketed by newly or previously established companies.

Beyond any doubts, robotic technologies will soon become fast, reliable, low-profile advanced instruments of everyday, routine OR use. I would like to draw attention to two caveats: (1) As the neurosurgical applications become more complex, rigorous safety tests and government and university regulations should be applied strictly to numerous small and bigger companies searching to establish their position in a new, fastgrowing market. (2) Similar to previous technologies, such as operating microscopes, 2-D and 3-D endoscopes, modern neuronavigation systems and intraoperative MRI, no technology will ever substitute surgical skill and sound surgical judgment and wisdom, as the old proverb goes "a fool with a tool is still a fool".

\section{Reference}

1. Lefranc M et al. (2014) Frameless robotic stereotactic biopsies: a consecutive series of 100 cases J Neurosurg (ahead of print) pages 1-11 